\title{
Osteoimmunology at the nexus of arthritis, osteoporosis, cancer, and infection
}

\author{
Dallas Jones, ${ }^{1,2}$ Laurie H. Glimcher, ${ }^{1,2,3}$ and Antonios O. Aliprantis ${ }^{1,2}$
}

${ }^{1}$ Department of Immunology and Infectious Diseases, Harvard School of Public Health, Boston, Massachusetts, USA. "2Department of Medicine, Division of Rheumatology, Allergy and Immunology, Brigham and Women's Hospital and Harvard Medical School, Boston, Massachusetts, USA. ${ }^{3}$ Ragon Institute of Massachusetts General Hospital, Harvard, and MIT, Boston, Massachusetts, USA.

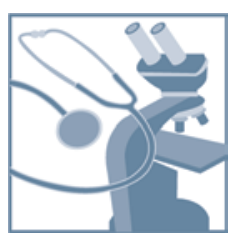

Over the past decade and a half, the biomedical community has uncovered a previously unappreciated reciprocal relationship between cells of the immune and skeletal systems. Work in this field, which has been termed "osteoimmunology," has resulted in the development of clinical therapeutics for seemingly disparate diseases linked by the common themes of inflammation and bone remodeling. Here, the important concepts and discoveries in osteoimmunology are discussed in the context of the diseases bridging these two organ systems, including arthritis, osteoporosis, cancer, and infection, and the targeted treatments used by clinicians to combat them.

\section{Introduction}

Advances in both medicine and basic science have elevated expectations that treatments for complex diseases will promptly emerge. However, the findings generated from these various disciplines have further emphasized the multifactorial nature of many diseases, which has hampered the progress toward remediation (1). This is especially true for diseases of the skeletal system that can manifest in individuals of any age, sex, or socioeconomic status as a result of genetic as well as environmental components (2). In a normal physiological state, the skeletal system provides support, mobility, protection for vital organs, and a mineral reservoir for calcium and phosphate. To achieve and adapt to these functions, the skeleton exists in a dynamic equilibrium characterized by continuous osteoclast-mediated bone resorption and osteoblast-mediated bone deposition (3). This biological process has been termed bone "remodeling" and occurs in coupled fashion with osteoblasts producing the key osteoclast differentiation factors and osteoclasts promoting bone formation via osteoblastic mediators they produce and liberate as they degrade bone. Osteoclasts differentiate from myeloid precursors in response to the cytokine RANKL and adhere to the bone surface where they secrete acid and proteolytic enzymes to degrade the inorganic and organic constituents of bone, respectively. Following the resorptive phase, mesenchymalderived osteoblasts migrate to the eroded area and initiate new bone formation through the secretion of an extracellular matrix consisting of both collagenous and noncollagenous proteins. It has become evident that both innate and adaptive immune cells exert profound effects on osteoclasts and osteoblasts through a panoply of cell-surface and secreted mediators (4). Moreover, an appreciation of the reciprocal influence of bone cells on immunity is emerging. Herein, the intersection of the immune and skeletal systems is discussed within the context of commonly encountered skeletal diseases. This review does not aim to provide a complete overview of osteoimmunology (for comprehensive review, see ref. 4) but rather to expose the reader to the key concepts, controversies,

Conflict of interest: Laurie H. Glimcher is a director of the Bristol-Myers Squibb Pharmaceutical Co. and holds equity therein. Laurie H. Glimcher is supported by a sponsored research grant from Merck and Co. Inc.

Citation for this article: J Clin Invest. 2011;121(7):2534-2542. doi:10.1172/JCI46262. and molecules in this emerging field as it relates to the major afflictions of the skeleton. Table 1 summarizes the clinical therapeutics mentioned in this review, and Figures 1 and 2 depict the molecules involved in crosstalk between immune and bone cells in the context of the diseases discussed here.

\section{Inflammatory arthritis - dysregulation of bone remodeling RANKL and IL-17 as key mediators}

The identification of RANKL and its intimate relationship to inflammation and bone destruction in RA launched the field of osteoimmunology over a decade ago. RANKL was originally cloned independently of its role in skeletal physiology as an immediate early gene induced in $\mathrm{T}$ cells after activation of $\mathrm{T}$ cell receptor signaling $(5,6)$. It was not until the RANKL-knockout mouse was generated and mutations were identified in human osteopetrotic patients that its function as the master regulator of osteoclastogenesis was realized (7-9). RANKL is a TNF family cytokine that can be produced by a number of different cell types including osteoblasts and fibroblasts in addition to T cells. Activation of the RANKL receptor (RANK) on mononuclear osteoclast precursors initiates a cascade of transcriptional changes culminating in multinucleated giant cell formation through cell fusion and the expression of the machinery needed for bone resorption including molecules needed for attachment to bone, acid secretion, and proteolysis. Interestingly, many of the transcription factors important for osteoclast differentiation are key regulators of immune responses, such as NF- $\mathrm{BB}$ and nuclear factor of activated $\mathrm{T}$ cells c1 (NFATc1); this finding was an early insight into the intimate relationship of skeletal biology and immunology. Indeed, genetic absence of $\mathrm{Nfatc1}$ in mice abrogates osteoclast differentiation in vivo and in vitro (10-12). The signaling and transcriptional mediators shared among immune cells and osteoclasts is an interesting molecular aspect of osteoimmunology that has been reviewed elsewhere (13). In addition to its effects on osteoclast precursors, RANKL has important roles in regulating immune processes such as lymph node organogenesis and self tolerance (14).

RA is a female-predominant autoimmune disease affecting approximately $1 \%$ of the population that results in chronic inflammation in the lining, or synovium, of peripheral diarthrodial joints (reviewed in ref. 15). Autoreactive T cells may initiate the inflam- 
Table 1

Clinical therapeutics targeting osteoimmunology pathways mentioned in this review

\begin{tabular}{|c|c|c|c|c|}
\hline Class & Examples & Indications ${ }^{A}$ & Mechanism in the context of osteoimmunology & Refs. \\
\hline Bisphosphonates & $\begin{array}{l}\text { Alendronate, } \\
\text { Risedronate, } \\
\text { Ibandronate, } \\
\text { Pamidronate, } \\
\text { Zoledronate }\end{array}$ & $\begin{array}{l}\text { Osteoporosis, cancer- } \\
\text { associated osteolysis }\end{array}$ & $\begin{array}{l}\text { Inhibit bone resorption by osteoclasts; } \\
\text { may cause ONJ through inhibition } \\
\text { of bone remodeling }\end{array}$ & 64,86 \\
\hline RANKL antagonists & Denosumab & $\begin{array}{l}\text { Osteoporosis, cancer- } \\
\text { associated osteolysis, RA }\end{array}$ & $\begin{array}{l}\text { Blocks bone resorption by preventing } \\
\text { RANKL mediated osteoclastogenesis }\end{array}$ & 20,127 \\
\hline TNF- $\alpha$ antagonists & $\begin{array}{l}\text { Etanercept, } \\
\text { Infliximab, } \\
\text { Adalimumab }\end{array}$ & RA & $\begin{array}{l}\text { Reduces inflammation; limits bone erosion } \\
\text { by decreasing RANKL-mediated osteoclastogenesis }\end{array}$ & 18,20 \\
\hline IL-1 antagonists & Anakinra & RA & $\begin{array}{l}\text { Reduces inflammation; limits bone erosion by } \\
\text { decreasing RANKL-mediated osteoclastogenesis }\end{array}$ & 18,20 \\
\hline IL-6 antagonists & Tocilizumab & RA & $\begin{array}{l}\text { Reduces inflammation; limits bone erosion by } \\
\text { decreasing RANKL-mediated osteoclastogenesis }\end{array}$ & 19,20 \\
\hline IL-17 antagonists & $\begin{array}{l}\text { LY2439821, } \\
\text { AIN457 }\end{array}$ & RA & $\begin{array}{l}\text { Reduces inflammation; limits bone erosion by } \\
\text { decreasing RANKL-mediated osteoclastogenesis }\end{array}$ & 36,37 \\
\hline IL-12/IL-23 antagonists & Ustekinumab & $\begin{array}{l}\text { Psoriasis, } \\
\text { psoriatic arthritis }\end{array}$ & $\begin{array}{l}\text { Reduces inflammation; limits bone erosion by } \\
\text { decreasing RANKL-mediated osteoclastogenesis }\end{array}$ & 38 \\
\hline Costimulation blockers & Abatacept & RA & $\begin{array}{l}\text { Disrupts costimulation of T cells by APCs; } \\
\text { may directly inhibit osteoclast formation }\end{array}$ & $18,20,46$ \\
\hline PTH agonists & Teriparatide & Osteoporosis & $\begin{array}{l}\text { Increases bone formation by stimulating the } \\
\text { production of anabolic Wnt proteins from T cells }\end{array}$ & 79 \\
\hline Estrogens & $\begin{array}{l}\text { Conjugated estrogen, } \\
\text { Estradiol }\end{array}$ & $\begin{array}{l}\text { Postmenopausal } \\
\text { osteoporosis }\end{array}$ & $\begin{array}{l}\text { Reduces bone resorption; Pathway that may involve } \\
\text { suppression of T cell derived pro-osteoclastic cytokines }\end{array}$ & $67-69$ \\
\hline
\end{tabular}

Alndications listed here are not necessarily approved by the FDA.

matory response, which is then perpetuated by autoantibody-producing $B$ cells and innate immune cells including macrophages that release proinflammatory cytokines such as TNF- $\alpha$, IL-1, and IL-6. Alternatively, disease initiation may arise from dysregulated innate immunity in response to environmental triggering of pattern recognition receptors (PRR) such as TLRs, leading to proinflammatory cytokines and activation of adaptive immunity. In either case, ultimately the normally thin synovial lining composed of fibroblast- and macrophage-like cells undergoes intense proliferation and angiogenesis to form a hyperplastic structure called the pannus. The pannus invades the joint space and destroys the cartilage through inflammatory cytokines that are directly toxic to chondrocytes and by degradative enzymes liberated from synoviocytes (16). Where the pannus tissue encroaches on periarticular bone, erosions occur through coupling of inflammation to osteoclast formation. Damage to articular cartilage and periarticular bone results in irreversible joint dysfunction and reduces quality of life (17). Knowledge of these basic mechanisms of RA pathogenesis has led to clinical approval of targeted biologic therapeutics that (a) block proinflammatory cytokines, (b) interrupt $\mathrm{T}$ cell costimulation, and (c) deplete B cells $(18,19)$.

Osteoclasts form where pannus tissue interfaces with periarticular bone and mediate bone erosions (reviewed in ref. 20). Data supporting this conclusion stem from studies where inflammatory arthritis was induced in rodents in either a genetically osteoclastdeficient background or concomitant with systemic administration of osteoprotegerin (OPG), a decoy receptor and inhibitor of RANKL (21-23). In each case, the rodents developed similar levels of inflammation, but demonstrated dramatically reduced periarticular bone destruction. Similar results have been reported in rodent models using high-potency bisphosphonates, such as zoledronic acid, which inhibit osteoclast function $(24,25)$. In humans, clinical trials of high potency bisphosphonates as well as denosumab, a monoclonal blocking antibody to RANKL approved for osteoporosis treatment, also strongly support the osteoclast as the primary mediator of bone erosion in RA $(26,27)$.

The source of pro-osteoclastogenic RANKL in RA has been an intense area of research, and the activated $T$ cell has emerged as a primary player through direct and indirect mechanisms (reviewed in ref. 28). A seminal paper published in 1999 was the first to link RANKL expression on T cells to osteoclastogenesis (23). In this study, TCR ligation was shown to induce expression of both membrane-bound and secreted RANKL, which supported osteoclastogenesis in vitro. Two additional studies confirmed the ability of activated human $\mathrm{T}$ cells to support in vitro osteoclastogenesis (29, 30). In contrast, a similarly timed publication suggested that $T$ cells exert antiosteoclastogenic activity through the ability of IFN- $\gamma$, a product of the Th1 subset, to promote degradation of a key signal transduction molecule downstream of the RANKL receptor (31). Consistent with this observation, while IFN- $\gamma$-positive T cells were shown to promote osteoclast differentiation in vitro via RANKL, their full osteoclastogenic potential was only realized when IFN- $\gamma$ activity was blocked (32). However, in vivo the effects of IFN- $\gamma$ on osteoclast differentiation may be context dependent, promoting bone loss in the setting of infection and estrogen withdrawal, as discussed in the sections below (33). Concomitant with these early 


\section{Rheumatoid arthritis}

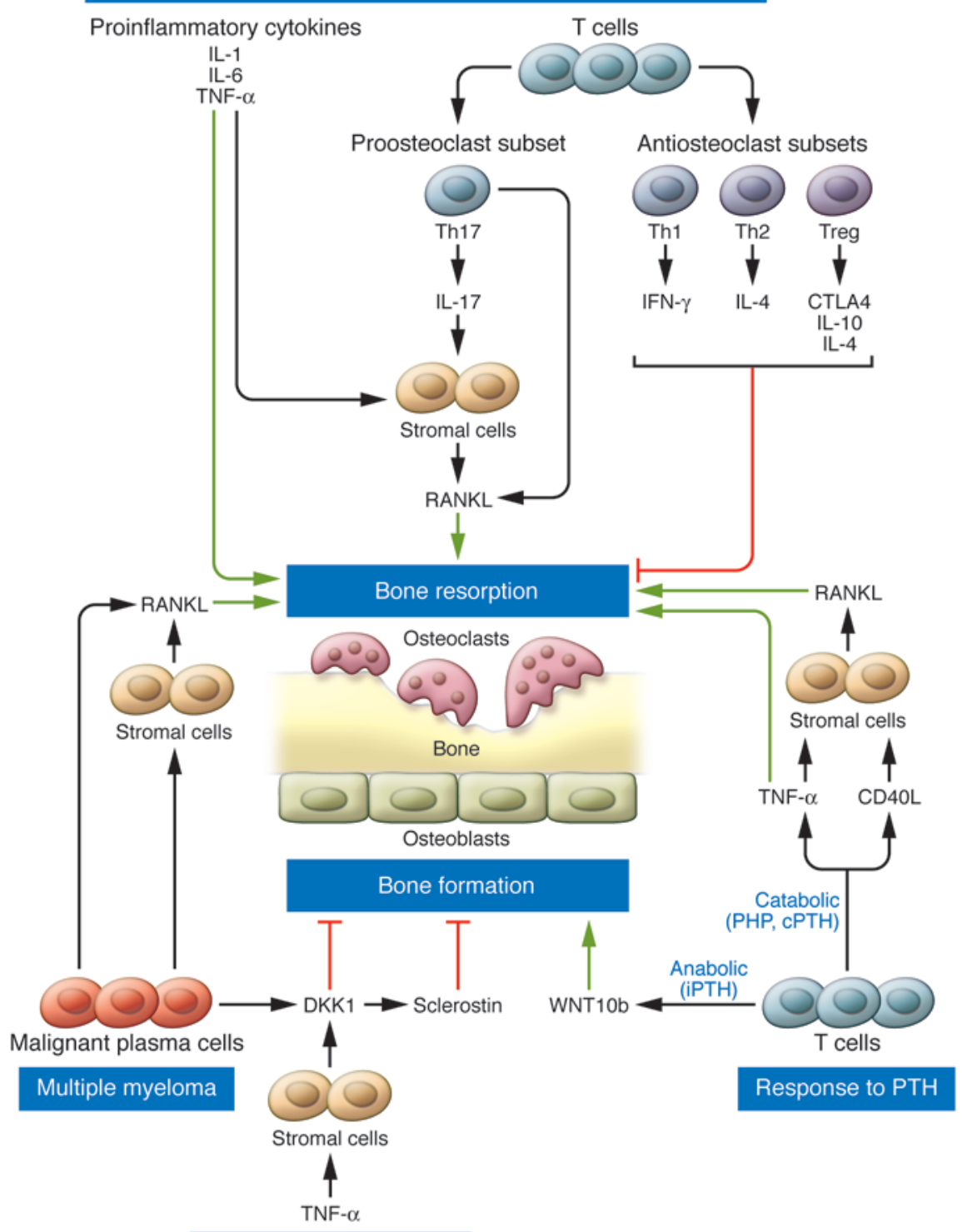

\section{Figure 1}

Schematic of the immune system's influence on skeletal remodeling. The effect of growth factors, cytokines, and cell-surface molecules generated by lymphoid populations on osteoclast-mediated bone resorption and osteoblast-mediated bone formation are depicted for physiologic and pathologic conditions. Red lines represent the repressive effects, and green arrows represent the stimulatory effects that the specific factor has on the target bone cell population. Stromal cells reflect various mesenchymal-derived cells such as osteoblastlineage cells and synovial fibroblasts. iPTH, intermittent PTH; cPTH, continuous PTH.

Rheumatoid arthritis

studies, Kotake et al. made the important observation that IL-17 was capable of driving osteoclast differentiation by inducing RANKL expression on osteoblasts (34). In addition, cells expressing both CD 4 and IL-17 were identified in RA synovium. This study not only implicated a new effector cytokine and $\mathrm{T}$ cell subset in this pathway (Th17 cells), but also an additional mechanism through which $\mathrm{T}$ cells could effect bone destruction: the induction of RANKL on mesenchymal-derived cells. Taken together, these early reports suggested that $\mathrm{T}$ cells influence osteoclast formation, but clarification of the details was needed.

A clearer picture emerged as the ability to manipulate $T$ helper subset differentiation in vitro matured. In a comprehensive study by Sato et al., Th 1 and Th 2 cells were both shown to inhibit osteoclast formation through their canonical cytokines IFN- $\gamma$ and IL-4, respectively (35). In contrast, Th17 cells were potent stimulators of osteoclastogenesis through IL-17. This effect was dependent on the presence of osteoblasts, though Th17 cells expressed membrane-bound RANKL, leading the authors to conclude that $T$ cell-derived RANKL is not sufficient for osteoclast differentiation. A tight correlation was found among the expression of IL-23 subunits (IL23A and IL12B), the upstream cytokine inducing Th17 differentiation, and RANKL in synovial biopsies from RA patients (35). Accordingly, biologic therapies inhibiting the IL-17 pathway by directly targeting this cytokine (LY2439821, AIN457) $(36,37)$ or blocking IL-23 (Ustekinumab) (38) are in clinical development for inflammatory arthritis.

In addition to $\mathrm{T}$ cells, the synovial fibroblast is a source of RANKL in RA. Synovial fibroblasts from RA patients express RANKL and support osteoclastogenesis in vitro $(39,40)$. In vivo, RANKL is expressed by synovial fibroblasts (41), with especially robust expression near the pannus-bone interface where erosions are found (42). The relative importance of RANKL derived from 
T cells, synovial fibroblasts, or osteoblast-like cells in RA has yet to be resolved and awaits definition.

Other mediators. A fourth $\mathrm{T}$ cell subset, the antiinflammatory Treg, inhibits osteoclast differentiation and function in vitro and suppresses inflammatory bone erosions in mice (43-45). Tregs negatively influence osteoclastogenesis through two mechanisms. The first involves cell contact and cytotoxic $\mathrm{T}$ lymphocyte antigen 4 (CTLA4), a membrane-bound molecule that blocks costimulation by APCs (43). In a T cell-independent model of RA generated by transgenic overexpression of TNF- $\alpha$ in mice, administration of CTLA4 moderately suppressed bone erosion, but had no influence on inflammation (46). The antierosive effect of abatacept, a CTLA4-Ig fusion protein approved for the treatment of RA, indicates a potential clinical relevance of this observation (47). Contact-independent production of cytokines by Tregs that negatively regulate osteoclast differentiation such as IL-4 and IL-10 is a second mechanism (48).

Clinical strategies utilizing inhibitors that target proinflammatory cytokines, such as TNF- $\alpha$, IL-6, and IL-1, have been approved for the treatment of RA $(18,19)$. These cytokines, which are produced by synovial fibroblasts and macrophages, promote bone destruction in RA by direct and indirect mechanisms. Indirectly, these cytokines augment RANKL expression in mesenchymal lineage cells $(20,34,49,50)$. They also directly act on osteoclast precursors to drive differentiation in synergy with RANKL (20, 51-53). Given the strong correlation between the activity of these cytokines on both inflammation and bone erosion, it is difficult to resolve which of these cytokines, if any, is the predominant driver of bone erosion in RA. In the inflamed joint, all may contribute.

Uncoupling bone resorption from formation in $R A$. Compared with physiologic bone turnover, a distinguishing feature of bone erosion in RA is the uncoupling of bone resorption from formation by osteoblasts (54). Two mechanisms underlying this observation have been defined. First, proinflammatory cytokines such as IL- 1 and TNF- $\alpha$ directly inhibit osteoblast differentiation and function (20). Second, soluble inhibitors of the Wingless (Wnt) pathway may suppress bone formation. In one recent study of a mouse model of RA, fewer cells expressing late osteoblast markers and reduced bone formation rates were found in bone adjacent to inflamed joints (55). Accordingly, increased levels of Wnt inhibitors were found in the inflamed synovial tissue. Diarra et al. showed that TNF- $\alpha$ upregulates the Wnt inhibitor Dickkopf-1 (DKK1) in synovial fibroblasts and that blocking antibodies to DKK1 could both promote bone formation, generating osteophytes around inflamed joints, and completely prevent bone erosions (56). DKK1 negatively regulates Wnt signaling by binding the Wnt coreceptors low-density lipoprotein receptor-related protein 5 (LRP5) and LRP6 (57). This finding was replicated in 3 models of RA, running the gamut from a $\mathrm{T}$ cell-dependent model of arthritis to one driven by transgenic TNF- $\alpha$ overexpression (56). Expression of DKK1 was documented in RA patients; serum levels positively correlated with disease activity and were suppressed by anti-TNF therapy. In contrast, patients with ankylosing spondylitis, another TNF- $\alpha$-dependent inflammatory arthropathy that unlike RA is characterized by periarticular new bone formation, had low serum DKK1 levels. The ability of DKK1 inhibition to reduce bone erosion and promote bone formation in RA may be related to the observation that Wnt pathway activation increases the expression of OPG and drives osteoblastic differentiation of periosteal cells, respectively $(56,58)$. In addition, inhibition of
DKK1 can also block TNF- $\alpha$-induced expression of another Wnt antagonist, Sclerostin, in osteoblasts (59). Future work will define whether activating the Wnt pathway can prevent erosion and promote bone healing in RA.

In summary, innate and adaptive immune cells in the environment of the inflamed joint promote destruction of periarticular bone through a complex network of factors that increase osteoclast activity and uncouple bone resorption from formation. Recent treatment advances in RA interfere with these pathways and preserve periarticular bone.

\section{Osteoimmunology at the interface of therapeutics for osteoporosis}

Estrogen, T cells, and bone mass. In healthy individuals, peak bone mass is achieved in early adulthood as a result of robust skeletal growth initiated at the onset of puberty (60). Subsequent to achieving this optimal level of bone mineral density, however, the skeleton in men and women exhibits an age-associated decline in bone mass (61). This progression toward osteoporosis is accelerated in postmenopausal women due to declining levels of estrogen, a hormone that exhibits osteoprotective properties through promoting osteoblast synthetic activity and retarding bone resorption by osteoclasts $(62,63)$. The realization of estrogen's central role in regulating postmenopausal bone mass has led to the development of multiple analogs clinically approved to treat osteoporosis (64). Interestingly, decreased estrogen levels might also contribute to the age-associated bone loss that is observed in elderly men (65). While estrogen can regulate skeletal remodeling by directly targeting osteoblasts and osteoclasts, it has also been reported to indirectly influence bone mass by targeting the immune system.

Estrogen has long been known to have an impact on lymphocyte physiology. However, the contribution of the adaptive immune system to the onset and progression of postmenopausal osteoporosis is disputed (66). Several studies have demonstrated that estrogen treatment modifies $\mathrm{T}$ cell production of cytokines and growth factors that promote bone resorption, including the proosteoclastic cytokines RANKL and TNF- $\alpha$ (67-69). T cells isolated from the bone marrow of postmenopausal women were shown to have elevated levels of RANKL when compared with those of either premenopausal women or postmenopausal women receiving hormone-replacement therapy. In contrast to its antiosteoclastogenic activity, described in the preceding section, in vivo IFN- $\gamma$ is necessary for ovariectomy-induced bone loss in mice and may drive osteoclast activity by promoting antigen-dependent $\mathrm{T}$ cell activation (33). Thus, the physiology of T cells may be altered following estrogen depletion and contribute to elevated bone resorption observed in postmenopausal women (70). In vivo analysis of bone loss in various lymphocyte-deficient mice following ovariectomy, a model that mimics the mechanisms through which bone loss occurs in postmenopausal osteoporosis, has led to conflicting findings $(67,71-74)$. While a number of factors may account for these differences, further studies will be required to conclusively determine what role, if any, the adaptive immune system plays in postmenopausal bone loss.

Parathyroid hormone acts on bone through $T$ cells. Recently, $\mathrm{T}$ cells have been demonstrated to have a more definitive role in mediating the bone-remodeling effects of parathyroid hormone (PTH). Under normal physiological conditions, PTH functions as a key regulator of calcium homeostasis. In response to hypocalcemia, PTH is released by the parathyroid gland and raises blood calcium 
levels in part through stimulating bone resorption. Calcium that is stored in the skeleton is released into the circulation and suppresses further production of PTH by the parathyroid gland. In certain pathological conditions, such as primary hyperparathyroidism (PHP), PTH is produced at supraphysiological levels, leading to accelerated bone loss and an increased incidence of fracture (75).

Continuous infusion of PTH in rodents also leads to decreased bone mass through augmenting osteoclast activity, thereby mimicking the catabolic effects of PTH observed in PHP. A recent analysis of several strains of genetically modified mice revealed that $\mathrm{T}$ cells play a role in promoting increased bone resorption observed with continuous PTH administration $(76,77)$. These studies showed that PTH signals through PTH receptor 1 on bone marrow T cells to augment production of TNF- $\alpha$ (77). While TNF- $\alpha$ exerts pro-osteoclastic activity by targeting osteoclast progenitors, the production of TNF- $\alpha$ by T cells was surprisingly shown to induce the upregulation of CD40 on the cell surface of the bone marrow stromal cells. The CD40 ligand-expressing T cells engage and induce signaling through CD40 on the stromal cells, which results in augmented RANKL production and downregulated OPG by this population, thus providing an environment conducive to osteoclastogenesis (76).

While continuous infusion of PTH leads to reduced bone mass through elevated bone resorption, paradoxically, intermittent administration of PTH through daily injections produces an anabolic effect that promotes bone formation through stimulation of osteoblast proliferation and function (78). Because of this latter effect, intermittent PTH infusion is the only FDA-approved anabolic modality for the treatment of osteoporosis (79). Using a series of $\mathrm{T}$ cell-deficient mice, it was demonstrated that the anabolic effects of PTH are blunted in the absence of this lymphocyte population. Thus, like their role in the catabolic effects of PTH, $\mathrm{T}$ cells appear to have a role in the anabolic effects of PTH (76). A series of additional experiments found that the $\mathrm{CD} 8^{+} \mathrm{T}$ cell population within the bone marrow is responsible for enhancing the bone anabolic effects of PTH through the production of Wnt10b. As mentioned above, the combination of both human and mouse genetics has demonstrated the central role that Wnt signaling has in regulating bone mass accrual (80). Therefore, compounds that target this pathway could provide a valuable therapeutic approach to preventing bone loss through promoting anabolic bone formation. While PTH can also promote bone formation through mechanisms that are $\mathrm{T}$ cell independent, the contribution of $\mathrm{T}$ cells in promoting the anabolic effects of PTH underscores the importance of gaining a greater understanding of the function that the adaptive immune system has in regulating skeletal remodeling to identify additional therapeutic targets.

\section{Cancer and the skeletal system}

Lytic bone lesions. In addition to providing mobility and protection for vital organs, the skeleton also serves as an essential environment in which hematopoiesis can occur. The stromal cells that reside within the bone marrow and line the endosteal surfaces provide a niche rich in growth factors, cytokines, and adhesion molecules that sustains the pluripotent state and self renewal of hematopoietic stem cells $(81,82)$. However, the fertile microenvironment within the bone marrow can also provide a niche for the development and proliferation of certain neoplastic diseases, including those of hematological origin that arise within the marrow as well as metastatic solid tumors (83). Often, the presence of these malignancies has an adverse effect on the skeleton that commonly manifests as osteolytic lesions causing patients to present with pain, pathologic fractures, hypercalcemia, spinal cord compression, and inexorable decline in mobility and quality of life. A greater understanding of the molecular mechanisms that maintain the balance of bone formation and bone resorption during normal skeletal remodeling has led to the identification of novel therapeutics such as bisphosphonates and denosumab for the treatment of the pathological bone loss associated with these diseases (84-86).

Regulation of bone turnover by myeloma cells. Multiple myeloma (MM) arises from the clonal expansion of malignant plasma cells within the bone marrow and is often associated with adverse skeletal events. Up to $80 \%$ of individuals diagnosed with myeloma present with skeletal pathologies over the course of the disease (87). Furthermore, the degree of bone destruction caused by these malignant plasma cells leads to a marked elevation in the incidence of fracture (88). Myelomas are the only skeletal malignancy that are purely lytic and exhibit potent osteolytic capacity through their ability to promote bone resorption by secreting a myriad of cytokines and chemokines as well as altering the RANKL/OPG ratio within the marrow microenvironment to favor osteoclastogenesis (89-92). Given the high degree of osteoclast-mediated bone destruction associated with MM, bisphosphonates are routinely incorporated into treatment regimens. Studies indicate that bisphosphonates are capable of preventing further bone loss in patients with MM and thus decrease both the number of skeletal-related events and the amount of bone pain reported by patients $(93,94)$. Interestingly, bisphosphonates have also been reported to have antimyeloma effects in part through activating $\gamma \delta$ T cells, which possess cytotoxic antitumor properties (95). Therefore, bisphosphonate treatment of individuals with MM may decrease tumor burden as well as reduce the number of osteolytic events. However, given the potential adverse effects of prolonged bisphosphonate use, additional therapies to combat the osteolytic potential of MM are needed. In recent clinical trials, denosumab has been shown to reduce both markers of bone resorption and the number of skeletal-related events in patients with $\mathrm{MM}$, suggesting that this therapy could provide a needed alternative for MM patients $(84,85)$.

In addition to producing factors that promote bone resorption, MM cells augment the rate of bone destruction by producing factors such as DKK1, which suppress new formation through negatively regulating Wnt signaling $(57,96)$. DKK1 secreted by these malignant cells binds to the LRP5/ 6 coreceptors on the surface of osteoprogenitor cells, prohibiting their proliferation and differentiation. Preclinical studies in murine models of MM have found that neutralizing antibodies to DKK1 are capable of increasing bone volume through augmenting osteoblast numbers and serum osteocalcin levels (97). Thus, discovery of novel anabolic agents that promote bone formation through stimulating Wnt signaling could provide an alternative treatment of MM-induced osteolysis.

RANK/RANKL signaling in tumorigenesis and metastasis. As described above, RANK/RANKL signaling is essential for osteoclast formation and bone resorption that occurs during skeletal remodeling. Analysis of Rank-null mice also uncovered an unexpected role for this signaling axis in the development of the lactating mammary glands during pregnancy (98). While physiological levels of RANK/ RANKL signaling stimulate the proliferation and cell survival of mammary epithelial cells, aberrant RANK/RANKL signaling in these tissues has recently been shown to influence the onset and progression of progestin-mediated breast tumorigenesis $(99,100)$. 
A

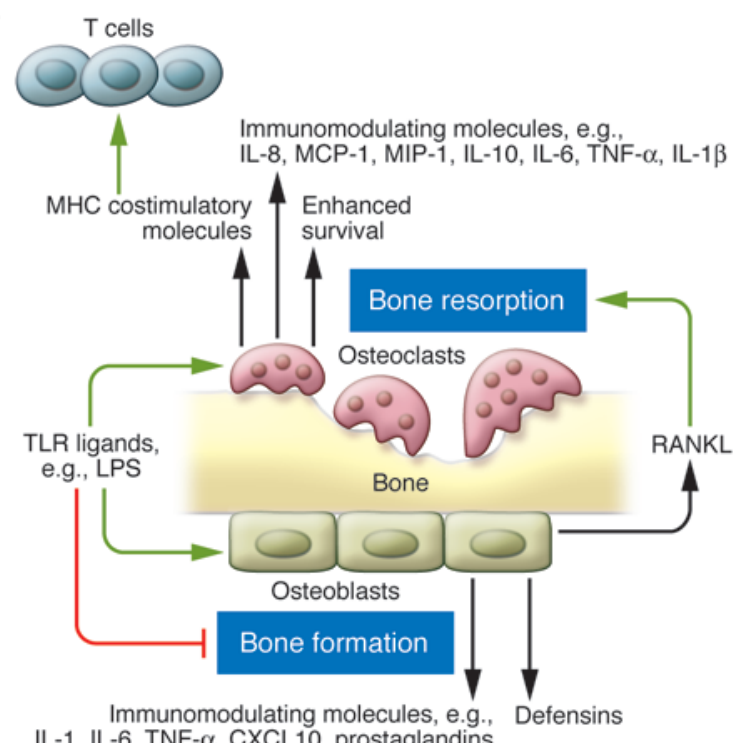

IL-1, IL-6, TNF- $\alpha$, CXCL10, prostaglandins

B

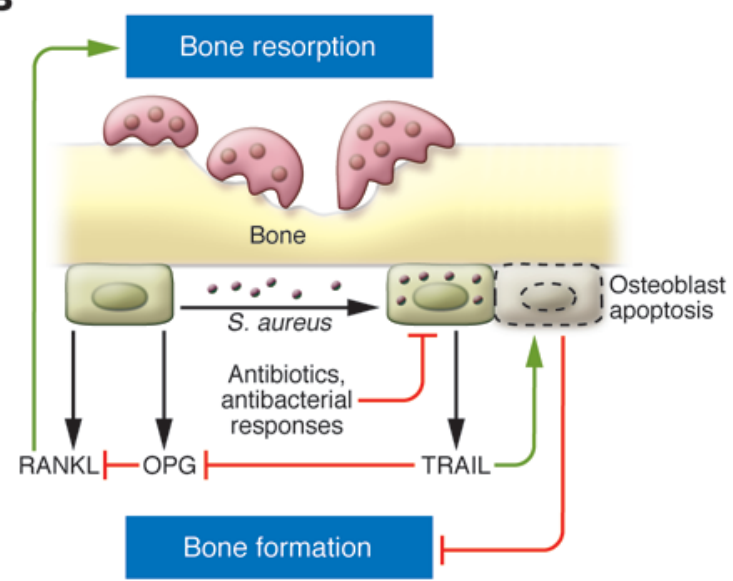

C

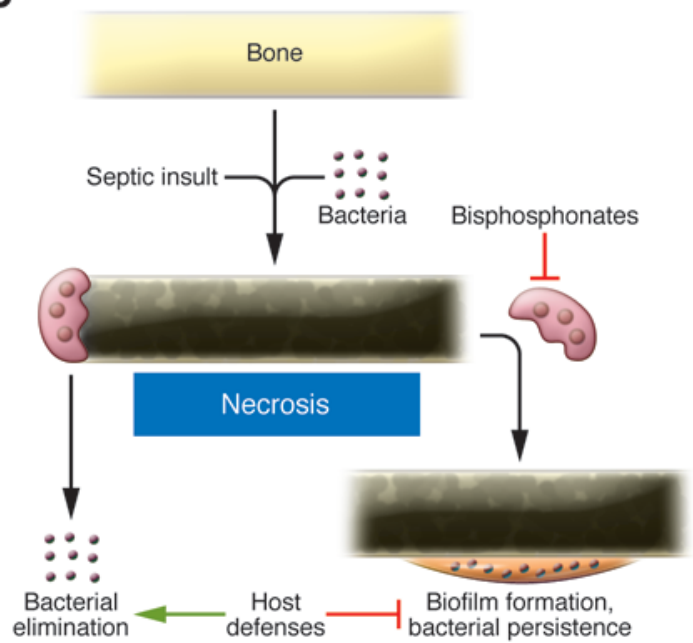

\section{Figure 2}

Potential roles of osteoclasts and osteoblasts in host defense against bacterial pathogens. (A) TLR ligands have multiple effects on osteoblasts and osteoclasts, eliciting responses that modulate inflammation and immunity, promote bacterial killing, and shift the balance of bone remodeling toward resorption. (B) Infection of osteoblasts with $S$. aureus may provide the bacteria with a niche to avoid antibiotics and antibacterial responses and promote bone destruction through TRAIL, which can sequester OPG and induce osteoblast apoptosis. (C) A potential mechanism for bisphosphonate ONJ. A septic insult to bone such as a tooth extraction results in necrosis. Normally the necrotic bone is removed by osteoclasts. When osteoclast activity is blocked by bisphosphonates, bacteria produce biofilms on the necrotic tissue allowing them to evade host defense mechanisms.

It was demonstrated that progestin treatment of mice resulted in a robust induction of RANKL within the mammary gland. The upregulation of RANKL in this tissue promoted tumorigenesis by stimulating the proliferation of RANK-expressing mammary epithelial cells and protecting these cells from apoptosis in response to DNA damage. Blocking of RANK/RANKL signaling in mice through either genetic ablation of Rank or by treating mice with RANKL-Fc abrogated the onset of mammary tumors. These findings suggest that targeting this pathway with therapies such as denosumab may provide a novel treatment for breast cancer.

Therapies that block RANK/RANKL signaling may also decrease the ability of osteotropic tumors to successfully metastasize to bone. Signaling through RANK on the surface of human epithelial tumor cells as well as melanoma cells has been shown to induce a chemotactic response in these tumor cells. In a murine model of melanoma metastasis, therapeutic treatment of mice with OPG to neutralize RANKL significantly reduced the tumor burden within the bones but not other organs (101). The authors of this paper concluded that RANKL present in the bone microenvironment could function as a "soil factor" allowing for successful engraftment of metastasizing tumors.

\section{Osteoclasts and osteoblasts in host defense against infection}

The interaction of bone cells with bacteria. Some of the evolutionary pressure driving the intimate relationship of the immune and skeletal systems likely arose from the need to combat bone infections. Bone infection, or osteomyelitis (OM), arises from either hematogenous spread or direct local extension of bacteria and presents acutely or as chronic infection. In adults, Staphylococcus aureus is the most common pathogen in OM (102). Lengthy courses of antibiotics are needed with or without surgical debridement, but antibiotic resistance and bacterial biofilm formation make eradication difficult and recurrences are frequent (103). Areas of bone resorption (osteolysis) surrounded by bone formation (osteosclerosis and periosteal new bone formation) are the radiographic characteristics of OM. The ability of the inflamed environment in $\mathrm{OM}$ or chronic periodontal disease to induce bone resorption by osteoclasts has been reviewed elsewhere (104), and many of the mechanisms driving bone loss in RA likely contribute to these conditions as well. Here, we focus on how the osteoclast and osteoblast participate in antibacterial host defense (Figure 2, A-C).

The initial response to a bacterial challenge is mediated by innate PRRs, such as TLRs that identify molecular patterns unique to microbes (105). In osteoblasts and osteoclasts, TLR 
ligands have diverse effects (reviewed in ref. 106). For example, stimulation of osteoblasts with the TLR4 ligand lipopolysaccharide (LPS) increases the expression of RANKL as well as proinflammatory molecules, including cytokines (IL-1, IL-6, TNF- $\alpha$ ), chemokines (CXLC10), and prostaglandins, which could serve to promote bone resorption and incite inflammation (Figure 2A and refs. 102, 106-111). Furthermore, LPS inhibits osteoblast differentiation and mineralization, contributing to the imbalance in bone remodeling seen in OM (112).

Interesting data indicate that osteoblasts can be directly infected with $S$. aureus, classically thought of as an extracellular pathogen (Figure 2B) (113). Though not formally proven, this has been proposed as a mechanism by which pathogens causing OM evade host responses and antibiotics (113). Furthermore, infection of osteoblasts with $S$. aureus promotes production of TNF-related apoptosis-inducing ligand (TRAIL), which can tip the bone remodeling scale toward resorption by inducing osteoblast apoptosis (114, 115 ) and sequestering OPG, resulting in higher RANKL availability $(102,113)$. Last, some evidence exists that osteoblasts make antibacterial peptides $(116,117)$. Documentation of the physiologic relevance of this observation and whether osteoblasts kill bacteria is lacking, but could be clinically important.

Osteoclasts also respond to TLR ligands (Figure 2A). Stimulation of osteoclast precursors with LPS inhibits RANKL-induced differentiation, likely by decreasing RANK levels $(118,119)$. In contrast, exposure after initiation of the differentiation program augments osteoclastogenesis and promotes survival $(106,118)$. In vivo, administration of sublethal doses of LPS induces systemic bone loss through an incompletely characterized IFN- $\gamma$-dependent pathway (33). Osteoclasts also produce proinflammatory mediators, such as IL-8, MCP-1, and MIP-1, after TLR stimulation $(120,121)$. A recent study showed that LPS stimulation of human osteoclasts resulted in IL-10, IL- 6 , TNF- $\alpha$, and IL- $1 \beta$ production as well as increased expression of MHC and costimulatory molecules important for antigen presentation (122). Accordingly, osteoclasts internalized soluble antigen, processed it, and presented it to $\mathrm{T}$ cells to induce proliferative and cytokine responses.

Osteoclast blockade and osteonecrosis of the jaw. In vivo, inhibition of osteoclast activity by bisphosphonates is associated with osteonecrosis of the jaw (ONJ), a condition characterized by devitalized bone with overlying soft tissue defects and concomitant chronic infection. ONJ most commonly occurs after dental trauma in patients treated with high-potency bisphosphonates (123). In a mouse model of OM, inhibition of osteoclasts by either bisphosphonates or excess OPG promoted abscess formation and increased bacterial burden (124). This may be due to failure of osteoclasts to remove necrotic bone resulting in a niche for invad- ing bacteria to establish pathogenic biofilms (Figure 2C). Another recent publication showed that dental extraction in normal mice results in bone necrosis surrounding the extraction socket, followed by angiogenesis and bone remodeling to remove the dead bone. In mice treated with bisphosphonates, angiogenesis and remodeling were blocked and necrotic bone was retained (125). It will be important to follow whether postmarketing surveillance of new antiosteoclast agents, such as denosumab (126), reveals an association of these drugs with ONJ.

Future work will examine the role of individual TLRs and other PRRs in osteoclasts and osteoblasts in models of OM and ONJ. Whether manipulation of host defense pathways in these cells can augment innate bone immunity and help eradicate treatmentresistant infections deserves further exploration.

\section{Concluding remarks and future directions}

An understanding of the connection between immune cells and osteoclasts and osteoblasts, and the mediators through which these cells communicate, has led to the development of therapeutics aimed at disrupting this often pathologic interaction. Over the next five to ten years, investigators will better define the clinical utility of new antiresorptive and anabolic agents in the treatment of skeletal diseases. Moreover, whether molecules targeting the Wnt pathway can augment bone formation while simultaneously suppressing resorption in humans will be tested. Last, while the majority of osteoimmunology studies have focused on how immune cells have an impact on skeletal remodeling, it is likely that osteoblasts and osteoclasts reciprocally regulate immune responses. A better understanding of this aspect of the osteoimmunology relationship could pave the way for unique strategies to control inflammation and bone loss in disease.

\section{Acknowledgments}

Antonios O. Aliprantis holds a Career Award for Medical Scientists from the Burroughs Wellcome Fund and is supported by award number K08AR054859 from the National Institute of Arthritis and Musculoskeletal and Skin Diseases. This work was also supported by NIH grants HD055601 (to L.H. Glimcher) and K99AR055668 (to D. Jones). The content is solely the responsibility of the authors and does not necessarily represent the official views of the National Institute of Arthritis and Musculoskeletal and Skin Diseases or the NIH.

Address correspondence to: Antonios O. Aliprantis, Harvard School of Public Health, 651 Huntington Avenue, FXB 205, Boston, Massachusetts 02115, USA. Phone: 617.432.4867; Fax: 617.432.0084; E-mail: aaliprantis@partners.org.
1. Zelzer E, Olsen BR. The genetic basis for skeletal diseases. Nature. 2003;423(6937):343-348.

2. Zaidi M. Skeletal remodeling in health and disease. Nat Med. 2007;13(7):791-801.

3. Karsenty G, Wagner EF. Reaching a genetic and molecular understanding of skeletal development. Dev Cell. 2002;2(4):389-406.

4. Takayanagi H. Osteoimmunology and the effects of the immune system on bone. Nat Rev Rheumatol. 2009;5(12):667-676.

5. Wong BR, et al. TRANCE, a new tumor necrosis factor (TNF) family member predominantly expressed in T cells, is a dendritic cell-specific survival factor. J Exp Med. 1997;186(1):1-6.

6. Wong BR, et al. TRANCE is a novel ligand of the tumor necrosis factor receptor family that acti- vates c-Jun N-terminal kinase in T cells. J Biol Chem. 1997;272(40):25190-25194.

7. Kong YY, et al. OPGL is a key regulator of osteoclastogenesis, lymphocyte development and lymph-node organogenesis. Nature. 1999;397(6717):315-323.

8. Kim N, Odgren PR, Kim DK, Marks SC Jr, Choi Y. Diverse roles of the tumor necrosis factor family member TRANCE in skeletal physiology revealed by TRANCE deficiency and partial rescue by a lymphocyte-expressed TRANCE transgene. Proc Natl Acad Sci U S A. 2000;97(20):10905-10910.

9. Villa A, Guerrini MM, Cassani B, Pangrazio A, Sobacchi C. Infantile malignant, autosomal recessive osteopetrosis: the rich and the poor. Calcif Tissue Int. 2009;84(1):1-12.

10. Asagiri M, et al. Autoamplification of NFATc1 expression determines its essential role in bone homeostasis. J Exp Med. 2005;202(9):1261-1269.

11. Aliprantis AO, et al. NFATc1 in mice represses osteoprotegerin during osteoclastogenesis and dissociates systemic osteopenia from inflammation in cherubism. J Clin Invest. 2008;118(11):3775-3789.

12. Takayanagi $\mathrm{H}$, et al. Induction and activation of the transcription factor NFATc1 (NFAT2) integrate RANKL signaling in terminal differentiation of osteoclasts. Dev Cell. 2002;3(6):889-901.

13. Negishi-Koga T, Takayanagi H. Ca2+-NFATc1 signaling is an essential axis of osteoclast differentiation. Immunol Rev. 2009;231(1):241-256.

14. Leibbrandt A, Penninger JM. Novel functions of RANK(L) signaling in the immune system. Adv Exp Med Biol. 2010;658:77-94. 
15. Sweeney SE, Firestein GS. Rheumatoid arthritis: regulation of synovial inflammation. Int J Biochem Cell Biol. 2004;36(3):372-378.

16. Noss EH, Brenner MB. The role and therapeutic implications of fibroblast-like synoviocytes in inflammation and cartilage erosion in rheumatoid arthritis. Immunol Rev. 2008;223:252-270.

17. Heller JE, Shadick NA. Outcomes in rheumatoid arthritis: incorporating the patient perspective. Curr Opin Rheumatol. 2007;19(2):101-105.

18. Singh JA, et al. Biologics for rheumatoid arthritis: an overview of Cochrane reviews. Cochrane Database Syst Rev. 2009; (4):CD007848.

19. Singh JA, Beg S, Lopez-Olivo MA. Tocilizumab for rheumatoid arthritis. Cochrane Database Syst Rev. 2010;(7):CD008331.

20. Karmakar S, Kay J, Gravallese EM. Bone damage in rheumatoid arthritis: mechanistic insights and approaches to prevention. Rheum Dis Clin North Am. 2010;36(2):385-404.

21. Pettit AR, et al. TRANCE/RANKL knockout mice are protected from bone erosion in a serum transfer model of arthritis. Am J Pathol. 2001; 159(5):1689-1699.

22. Redlich K, et al. Osteoclasts are essential for TNF $\alpha$ mediated joint destruction. J Clin Invest. 2002; 110(10):1419-1427.

23. Kong YY, et al. Activated $\mathrm{T}$ cells regulate bone loss and joint destruction in adjuvant arthritis through osteoprotegerin ligand. Nature. 1999; 402(6759):304-309.

24. Sims NA, et al. Targeting osteoclasts with zoledronic acid prevents bone destruction in collagen-induced arthritis. Arthritis Rheum. 2004;50(7):2338-2346.

25. Herrak P, et al. Zoledronic acid protects against local and systemic bone loss in tumor necrosis factor-mediated arthritis. Arthritis Rheum. 2004; 50(7):2327-2337.

26. Jarrett SJ, et al. Preliminary evidence for a structural benefit of the new bisphosphonate zoledronic acid in early rheumatoid arthritis. Arthritis Rheum. 2006;54(5):1410-1414.

27. Cohen SB, et al. Denosumab treatment effects on structural damage, bone mineral density, and bone turnover in rheumatoid arthritis: a twelve-month, multicenter, randomized, double-blind, placebocontrolled, phase II clinical trial. Arthritis Rheum. 2008;58(5):1299-1309.

28. Adamopoulos IE, Bowman EP. Immune regulation of bone loss by Th17 cells. Arthritis Res Ther. 2008;10(5):225.

29. Horwood NJ, Kartsogiannis V, Quinn JM, Romas E, Martin TJ, Gillespie MT. Activated T lymphocytes support osteoclast formation in vitro. Biochem Biophys Res Commun. 1999;265(1):144-150.

30. Kotake S, et al. Activated human T cells directly induce osteoclastogenesis from human monocytes: possible role of $\mathrm{T}$ cells in bone destruction in rheumatoid arthritis patients. Arthritis Rheum. 2001; 44(5):1003-1012.

31. Takayanagi $\mathrm{H}$, et al. T-cell-mediated regulation of osteoclastogenesis by signalling cross-talk between RANKL and IFN- $\gamma$. Nature. 2000;408(6812):600-605

32. Kotake $\mathrm{S}$, et al. IFN- $\gamma$-producing human $\mathrm{T}$ cells directly induce osteoclastogenesis from human monocytes via the expression of RANKL. Eur J Immunol. 2005;35(11):3353-3363.

33. Gao Y, et al. IFN- $\gamma$ stimulates osteoclast formation and bone loss in vivo via antigen-driven $\mathrm{T}$ cell activation. J Clin Invest. 2007;117(1):122-132.

34. Kotake S, et al. IL-17 in synovial fluids from patients with rheumatoid arthritis is a potent stimulator of osteoclastogenesis. J Clin Invest. 1999; 103(9):1345-1352.

35. Sato K, et al. Th17 functions as an osteoclastogenic helper $\mathrm{T}$ cell subset that links $\mathrm{T}$ cell activation and bone destruction. J Exp Med. 2006; 203(12):2673-2682.
36. Hueber W, et al. Effects of AIN457, a fully human antibody to interleukin-17A, on psoriasis, rheumatoid arthritis, and uveitis. Sci Transl Med. 2010; 2(52):52ra72

37. Genovese MC, et al. LY2439821, a humanized anti-interleukin-17 monoclonal antibody, in the treatment of patients with rheumatoid arthritis: A phase I randomized, double-blind, placebo-controlled, proof-of-concept study. Arthritis Rheum. 2010; 62(4):929-939.

38. Rozenblit M, Lebwohl M. New biologics for psoriasis and psoriatic arthritis. Dermatol Ther. 2009;22(1):56-60.

39. Takayanagi $\mathrm{H}$, et al. Involvement of receptor activator of nuclear factor- $\kappa \mathrm{B}$ ligand/osteoclast differentiation factor in osteoclastogenesis from synoviocytes in rheumatoid arthritis. Arthritis Rheum. 2000; 43(2):259-269.

40. Takayanagi $\mathrm{H}$, et al. A new mechanism of bone destruction in rheumatoid arthritis: synovial fibroblasts induce osteoclastogenesis. Biochem Biophys Res Commun. 1997;240(2):279-286.

41. Gravallese EM, et al. Synovial tissue in rheumatoid arthritis is a source of osteoclast differentiation factor. Arthritis Rheum. 2000;43(2):250-258.

42. Pettit AR, Walsh NC, Manning C, Goldring SR, Gravallese EM. RANKL protein is expressed at the pannus-bone interface at sites of articular bone erosion in rheumatoid arthritis. Rheumatology (Oxford). 2006;45(9):1068-1076.

43. Zaiss MM, et al. Treg cells suppress osteoclast formation: a new link between the immune system and bone. Arthritis Rheum. 2007;56(12):4104-4112.

44. Zaiss MM, et al. Regulatory $\mathrm{T}$ cells protect from local and systemic bone destruction in arthritis. JImmunol. 2010;184(12):7238-7246.

45. Kelchtermans H, Geboes L, Mitera T, Huskens D, Leclercq G, Matthys P. Activated CD4+CD25+ regulatory $\mathrm{T}$ cells inhibit osteoclastogenesis and collagen-induced arthritis. Ann Rheum Dis. 2009; 68(5):744-750.

46. Axmann R, et al. CTLA-4 directly inhibits osteoclast formation. Ann Rheum Dis. 2008;67(11):1603-1609.

47. Kremer JM, et al. Results of a two-year followup study of patients with rheumatoid arthritis who received a combination of abatacept and methotrexate. Arthritis Rheum. 2008;58(4):953-963.

48. Kim YG, Lee CK, Nah SS, Mun SH, Yoo B, Moon HB. Human CD 4+CD25+ regulatory T cells inhibit the differentiation of osteoclasts from peripheral blood mononuclear cells. Biochem Biophys Res Commun. 2007;357(4):1046-1052.

49. Tunyogi-Csapo M, et al. Cytokine-controlled RANKL and osteoprotegerin expression by human and mouse synovial fibroblasts: fibroblast-mediated pathologic bone resorption. Arthritis Rheum. 2008; 58(8):2397-2408.

50. Hashizume M, Hayakawa N, Mihara M. IL-6 transsignaling directly induces RANKL on fibroblastlike synovial cells and is involved in RANKL induction by TNF $\alpha$ and IL-17. Rheumatology (Oxford). 2008;47(11):1635-1640.

51. Wei S, Kitaura H, Zhou P, Ross FP, Teitelbaum SL. IL-1 mediates TNF $\alpha$ osteoclastogenesis. J Clin Invest. 2005;115(2):282-290.

52. Lam J, Takeshita S, Barker JE, Kanagawa O, Ross FP, Teitelbaum SL. TNF $\alpha$ induces osteoclastogenesis by direct stimulation of macrophages exposed to permissive levels of RANK ligand. J Clin Invest. 2000;106(12):1481-1488.

53. Adamopoulos IE, et al. Interleukin-17A upregulates receptor activator of $\mathrm{NFKB}$ on osteoclast precursors. Arthritis Res Ther. 2010;12(1):R29.

54. Goldring SR. Bone and joint destruction in rheumatoid arthritis: what is really happening? J Rheumatol Suppl. 2002;65:44-48.

55. Walsh NC, et al. Osteoblast function is compromised at sites of focal bone erosion in inflammatory arthritis. J Bone Miner Res. 2009;24(9):1572-1585.

56. Diarra D, et al. Dickkopf- 1 is a master regulator of joint remodeling. Nat Med. 2007;13(2):156-163.

57. Semenov MV, Tamai K, Brott BK, Kuhl M, Sokol S, He X. Head inducer Dickkopf-1 is a ligand for Wnt coreceptor LRP6. Curr Biol. 2001;11(12):951-961.

58. Glass DA 2nd, et al. Canonical Wnt signaling in differentiated osteoblasts controls osteoclast differentiation. Dev Cell. 2005;8(5):751-764.

59. Heiland GR, et al. Neutralisation of Dkk-1 protects from systemic bone loss during inflammation and reduces sclerostin expression. Ann Rheum Dis. 2010;69(12):2152-2159.

60. Heaney RP, et al. Peak bone mass. Osteoporos Int. 2000;11(12):985-1009.

61. Riggs BL, Melton LJ 3rd. Involutional osteoporosis. NEngl J Med. 1986;314(26):1676-1686.

62. Riggs BL, Khosla S, Melton LJ 3rd. Sex steroids and the construction and conservation of the adult skeleton. Endocr Rev. 2002;23(3):279-302.

63. Syed F, Khosla S. Mechanisms of sex steroid effects on bone. Biochem Biophys Res Commun. 2005; 328(3):688-696.

64. Gass M, Dawson-Hughes B. Preventing osteoporosis-related fractures: an overview. Am J Med. 2006; 119(4 suppl 1):S3-S11.

65. Riggs BL, Khosla S, Melton LJ 3rd. A unitary model for involutional osteoporosis: estrogen deficiency causes both type I and type II osteoporosis in postmenopausal women and contributes to bone loss in aging men. J Bone Miner Res. 1998;13(5):763-773.

66. Pernis AB. Estrogen and CD4+ T cells. Curr Opin Rheumatol. 2007;19(5):414-420.

67. Cenci S, et al. Estrogen deficiency induces bone loss by enhancing T-cell production of TNF-alpha. J Clin Invest. 2000;106(10):1229-1237.

68. D'Amelio P, et al. Estrogen deficiency increases osteoclastogenesis up-regulating $\mathrm{T}$ cells activity: a key mechanism in osteoporosis. Bone. 2008; 43(1):92-100

69. Yoneda T, et al. Estrogen deficiency accelerates murine autoimmune arthritis associated with receptor activator of nuclear factor-kappa B ligandmediated osteoclastogenesis. Endocrinology. 2004; 145(5):2384-2391.

70. Eghbali-Fatourechi G, Khosla S, Sanyal A, Boyle WJ, Lacey DL, Riggs BL. Role of RANK ligand in mediating increased bone resorption in early postmenopausal women. J Clin Invest. 2003;111(8):1221-1230.

71. Yamaza T, et al. Pharmacologic stem cell based intervention as a new approach to osteoporosis treatment in rodents. PLoS One. 2008;3(7):e2615.

72. Pacifici R. T cells: critical bone regulators in health and disease. Bone. 2010;47(3):461-471.

73. Lee SK, et al. T lymphocyte-deficient mice lose trabecular bone mass with ovariectomy. J Bone Miner Res. 2006;21(11):1704-1712.

74. Anginot A, Dacquin R, Mazzorana M, Jurdic P. Lymphocytes and the Dap12 adaptor are key regulators of osteoclast activation associated with gonadal failure. PLoS One. 2007;2(7):e585.

75. Spiegel AM. Pathophysiology of primary hyperparathyroidism. J Bone Miner Res. 1991; 6(suppl 2):S15-S17.

76. Gao Y, et al. T cells potentiate PTH-induced cortical bone loss through CD40L signaling. Cell Metab. 2008;8(2):132-145.

77. Tawfeek H, et al. Disruption of PTH receptor 1 in T cells protects against PTH-induced bone loss. PLoS One. 2010;5(8):e12290.

78. Jilka RL. Molecular and cellular mechanisms of the anabolic effect of intermittent PTH. Bone. 2007;40(6):1434-1446.

79. File E, Deal C. Clinical update on teriparatide. Curr Rheumatol Rep. 2009;11(3):169-176.

80. Baron R, Rawadi G. Targeting the Wnt/beta-catenin pathway to regulate bone formation in the adult skeleton. Endocrinology. 2007;148(6):2635-2643. 
81. Calvi LM, et al. Osteoblastic cells regulate the haematopoietic stem cell niche. Nature. 2003 . 425(6960):841-846.

82. Zhang J, et al. Identification of the haematopoietic stem cell niche and control of the niche size. Nature. 2003;425(6960):836-841.

83. Iwasaki H, Suda T. Cancer stem cells and their niche. Cancer Sci. 2009;100(7):1166-1172.

84. Body JJ, et al. A study of the biological receptor activator of nuclear factor- $\mathrm{\kappa B}$ ligand inhibitor, denosumab, in patients with multiple myeloma or bone metastases from breast cancer. Clin Cancer Res. 2006; 12(4):1221-1228

85. Vij R, et al. An open-label, phase 2 trial of denosumab in the treatment of relapsed or plateau-phase multiple myeloma. Am J Hematol. 2009;84(10):650-656.

86. Costa L, Major PP. Effect of bisphosphonates on pain and quality of life in patients with bone metastases. Nat Clin Pract Oncol. 2009;6(3):163-174.

87. Callander NS, Roodman GD. Myeloma bone disease. Semin Hematol. 2001;38(3):276-285.

88. Melton LJ 3rd, Kyle RA, Achenbach SJ, Oberg AL, Rajkumar SV. Fracture risk with multiple myeloma: a population-based study. J Bone Miner Res. 2005; 20(3):487-493.

89. Choi SJ, et al. Macrophage inflammatory protein 1-alpha is a potential osteoclast stimulatory factor in multiple myeloma. Blood. 2000;96(2):671-675.

90. Giuliani N, Colla S, Morandi F, Rizzoli V. The RANK/RANK ligand system is involved in interleukin- 6 and interleukin-11 up-regulation by human myeloma cells in the bone marrow microenvironment. Haematologica. 2004;89(9):1118-1123.

91. Lee JW, et al. IL-3 expression by myeloma cells increases both osteoclast formation and growth of myeloma cells. Blood. 2004;103(6):2308-2315.

92. Standal T, et al. Osteoprotegerin is bound, internalized, and degraded by multiple myeloma cells. Blood. 2002;100(8):3002-3007.

93. Berenson JR, et al. Long-term pamidronate treatment of advanced multiple myeloma patient reduces skeletal events. Myeloma Aredia Study Group. J Clin Oncol. 1998;16(2):593-602.

94. Petcu EB, Schug SA, Smith H. Clinical evaluation of onset of analgesia using intravenous pamidronate in metastatic bone pain. J Pain Symptom Manage. 2002;24(3):281-284.

95. Mariani S, et al. Effector gammadelta T cells and tumor cells as immune targets of zoledronic acid in multiple myeloma. Leukemia. 2005;19(4):664-670.

96. Tian E, et al. The role of the Wnt-signaling antagonist DKK1 in the development of osteolytic lesions in multiple myeloma. N Engl J Med. 2003 ; 349(26):2483-2494.
97. Fulciniti M, et al. Anti-DKK1 mAb (BHQ880) as a potential therapeutic agent for multiple myeloma. Blood. 2009;114(2):371-379.

98. Fata JE, et al. The osteoclast differentiation factor osteoprotegerin-ligand is essential for mammary gland development. Cell. 2000;103(1):41-50.

99. Schramek D, et al. Osteoclast differentiation factor RANKL controls development of progestin-driven mammary cancer. Nature. 2010;468(7320):98-102.

100. Gonzalez-Suarez E, et al. RANK ligand mediates progestin-induced mammary epithelial proliferation and carcinogenesis. Nature. 2010;468(7320):103-107.

101.Jones DH, et al. Regulation of cancer cell migration and bone metastasis by RANKL. Nature. 2006; 440(7084):692-696.

102. Marriott I. Osteoblast responses to bacterial pathogens: a previously unappreciated role for boneforming cells in host defense and disease progression. Immunol Res. 2004;30(3):291-308.

103. Conterno LO, da Silva Filho CR. Antibiotics for treating chronic osteomyelitis in adults. Cochrane Database Syst Rev. 2009;(3):CD004439.

104. Graves DT, Li J, Cochran DL. Inflammation and uncoupling as mechanisms of periodontal bone loss. J Dent Res. 2011;90(2):143-153.

105. Kawai T, Akira S. The role of pattern-recognition receptors in innate immunity: update on Toll-like receptors. Nat Immunol. 2010;11(5):373-384.

106. Bar-Shavit Z. Taking a toll on the bones: regulation of bone metabolism by innate immune regulators. Autoimmunity. 2008;41(3):195-203.

107. Keeting PE, et al. Evidence for interleukin-1 $\beta$ production by cultured normal human osteoblast-like cells. J Bone Miner Res. 1991;6(8):827-833.

108.Ishimi Y, et al. IL- 6 is produced by osteoblasts and induces bone resorption. J Immunol. 1990; 145(10):3297-3303

109. Sakuma Y, et al. Impaired bone resorption by lipopolysaccharide in vivo in mice deficient in the prostaglandin E receptor EP4 subtype. Infect Immun. 2000;68(12):6819-6825

110.He J, Tomlinson R, Coon D, Gulati A, Cowan C. Proinflammatory cytokine expression in cyclooxygenase-2-deficient primary osteoblasts. J Endod. 2007;33(11):1309-1312.

111. Gasper NA, Petty CC, Schrum LW, Marriott I, Bost KL. Bacterium-induced CXCL10 secretion by osteoblasts can be mediated in part through toll-like receptor 4. Infect Immun. 2002;70(8):4075-4082.

112.Bandow K, et al. Molecular mechanisms of the inhibitory effect of lipopolysaccharide (LPS) on osteoblast differentiation. Biochem Biophys Res Commun. 2010;402(4):755-761.

113. Henderson B, Nair SP. Hard labour: bacterial infection of the skeleton. Trends Microbiol. 2003; 11(12):570-577.

114. Young AB, Cooley ID, Chauhan VS, Marriott I. Causative agents of osteomyelitis induce death domain-containing TNF-related apoptosis-inducing ligand receptor expression on osteoblasts. Bone. 2011;48(4):857-863.

115.Alexander EH, Rivera FA, Marriott I, Anguita J, Bost KL, Hudson MC. Staphylococcus aureus - induced tumor necrosis factor - related apoptosis - inducing ligand expression mediates apoptosis and caspase8 activation in infected osteoblasts. BMC Microbiol. 2003;3:5.

116. Varoga $\mathrm{D}$, et al. Osteoblasts participate in the innate immunity of the bone by producing human $\beta$-defensin-3. Histochem Cell Biol. 2009;131(2):207-218.

117. Varoga $D$, et al. The role of human $\beta$-defensin- 2 in bone. J Anat. 2008;213(6):749-757.

118. Takami M, Kim N, Rho J, Choi Y. Stimulation by toll-like receptors inhibits osteoclast differentiation. J Immunol. 2002;169(3):1516-1523.

119.Ji JD, et al. Inhibition of RANK expression and osteoclastogenesis by TLRs and IFN- $\gamma$ in human osteoclast precursors. J Immunol. 2009;183(11):7223-7233.

120. Rothe L, et al. Human osteoclasts and osteoclast-like cells synthesize and release high basal and inflammatory stimulated levels of the potent chemokine interleukin-8. Endocrinology. 1998;139(10):4353-4363.

121. Masuda T, Deng X, Tamai R. Mouse macrophages primed with alendronate down-regulate monocyte chemoattractant protein-1 (MCP-1) and macrophage inflammatory protein-1alpha (MIP-1alpha) production in response to Toll-like receptor (TLR) 2 and TLR4 agonist via Smad 3 activation. Int Immunopharmacol. 2009;9(9):1115-1121.

122. Li H, Hong S, Qian J, Zheng Y, Yang J, Yi Q. Cross talk between the bone and immune systems: osteoclasts function as antigen-presenting cells and activate CD4+ and CD8+ T cells. Blood. 2010;116(2):210-217.

123. Woo SB, Hellstein JW, Kalmar JR. Narrative [corrected] review: bisphosphonates and osteonecrosis of the jaws. Ann Intern Med. 2006;144(10):753-761.

124. Li D, et al. Effects of antiresorptive agents on osteomyelitis: novel insights into the pathogenesis of osteonecrosis of the jaw. Ann N Y Acad Sci. 2010;1192:84-94.

125. Bi Y, et al. Bisphosphonates cause osteonecrosis of the jaw-like disease in mice. Am J Pathol. 2010; 177(1):280-290.

126. Kendler DL, et al. Effects of denosumab on bone mineral density and bone turnover in postmenopausal women transitioning from alendronate therapy. J Bone Miner Res. 2010;25(1):72-81.

127. Miller PD. Denosumab: anti-RANKL antibody. Curr Osteoporos Rep. 2009;7(1):18-22. 